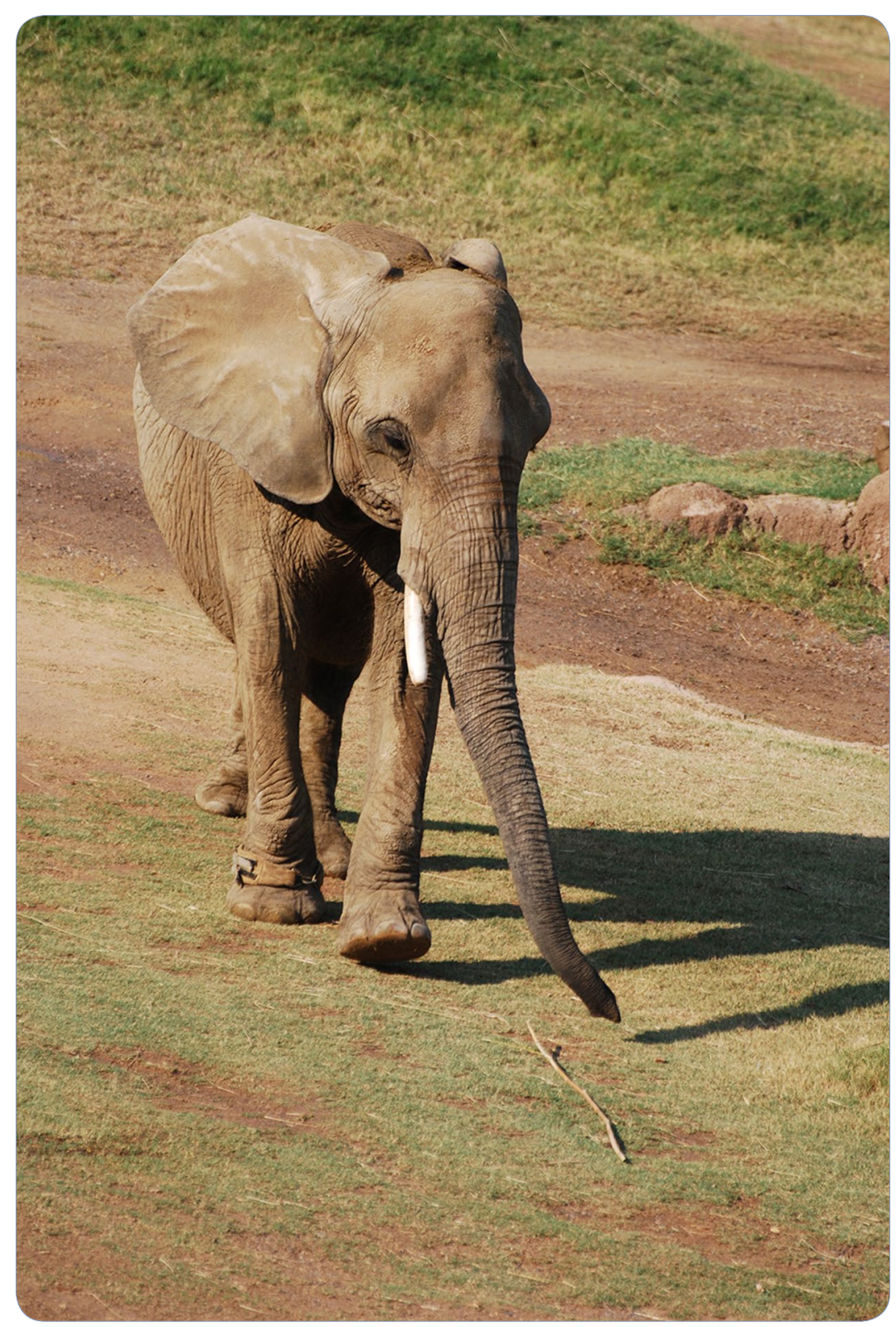

\title{
Using an active Radio Frequency Identification Real-Time Location System to remotely monitor animal movement in zoos
}

Scott et al. 


\title{
Using an active Radio Frequency Identification Real-Time Location System to remotely monitor animal movement in zoos
}

\author{
Nancy L. Scott ${ }^{1^{*}} \mathbb{D}$, Bernard Hansen ${ }^{2}$, Chase A. LaDue ${ }^{3} \mathbb{D}$, Carlson Lam ${ }^{4}$, Albert Lai ${ }^{4}$ and Lowell Chan ${ }^{4}$
}

\begin{abstract}
Background: Radio and satellite telemetry collars have been used across taxa, including with elephants, in situ for decades to collect data used for various analyses. To quantify the movement patterns of African elephants, Loxodonta africana, in a zoo, we modified a Real-Time Location System using active Radio Frequency Identification tags originally developed for inventory and asset tracking in warehouses. This is the first phase of a multi-phase project that we have termed the Real-Time Observer of Animal Movement. It allows for the continuous data collection and data analytics of elephant movement and space use in a mixed-species African savanna habitat at the Dallas Zoo. This system could prove to be a useful remote welfare tool for a variety of animal species at multiple facilities.

Results: We determined that a Real-Time Location System using Radio Frequency Identification tags could be used to continuously monitor elephant movement, social relationships, and exhibit use remotely over several acres in a zoo with a volume of data not otherwise possible to achieve. Real-time visual outputs include current location in the habitat, paths taken around the habitat, habitat preferences, social relationships between the elephants, and data tables with average rate of travel and distance traveled.

Conclusions: The pilot system at the Dallas Zoo is the first-ever use of active Radio Frequency Identification technology to observe zoo animal behavior patterns and monitor welfare. It has proven to be an effective tool for efficiently collecting data continuously and remotely throughout the day and night. It represents the first phase of a multifaceted project that will add capabilities and functionality for additional species and other zoos to form a network for sharing data to inform captive animal management strategies.
\end{abstract}

Keywords: Radio Frequency Identification, Real-Time Location System, Elephant, Animal welfare, Loxodonta africana, Zoo, RFID, RTLS

\section{Background}

The Dallas Zoo (DZ) in Dallas, Texas, was selected as the test site for Real-Time Observer of Animal Movement (ROAM) installation, the pilot system using active Radio Frequency Identification (RFID) tags to track the locomotion and exhibit use of African elephants (Loxodonta africana) in the award-winning mixed-species Giants of the Savanna exhibit, completed in 2010. The adult female elephants (aged 28-45 when the exhibit opened) have access

\footnotetext{
*Correspondence: nancy.scott@dallaszoo.com

${ }^{1}$ Conservation and Education, Dallas Zoo, 650 South RL Thornton Frwy, Dallas, TX 75203, USA

Full list of author information is available at the end of the article
}

to over 5 acres of visitor-viewable outdoor habitat including two large, naturalistic areas (Fig. 1). The larger space routinely used by the elephants, known as South Habitat, is connected directly to a large off-exhibit elephant barn. This habitat is readily observable by visitors from the interpretative center (known as Base Camp; Fig. 2) and two observation towers used exclusively by zoo personnel. North Habitat, the adjacent exhibit space, has one observation tower and contains giraffe (Giraffa camelopardalis), impala (Aepyceros melampus), zebra (Equus burchellii), ostrich (Struthio camelus), helmeted guinea fowl (Numida meleagris), vulturine guinea fowl (Acryllium vulturinum), and spur-winged geese (Plectropterus 


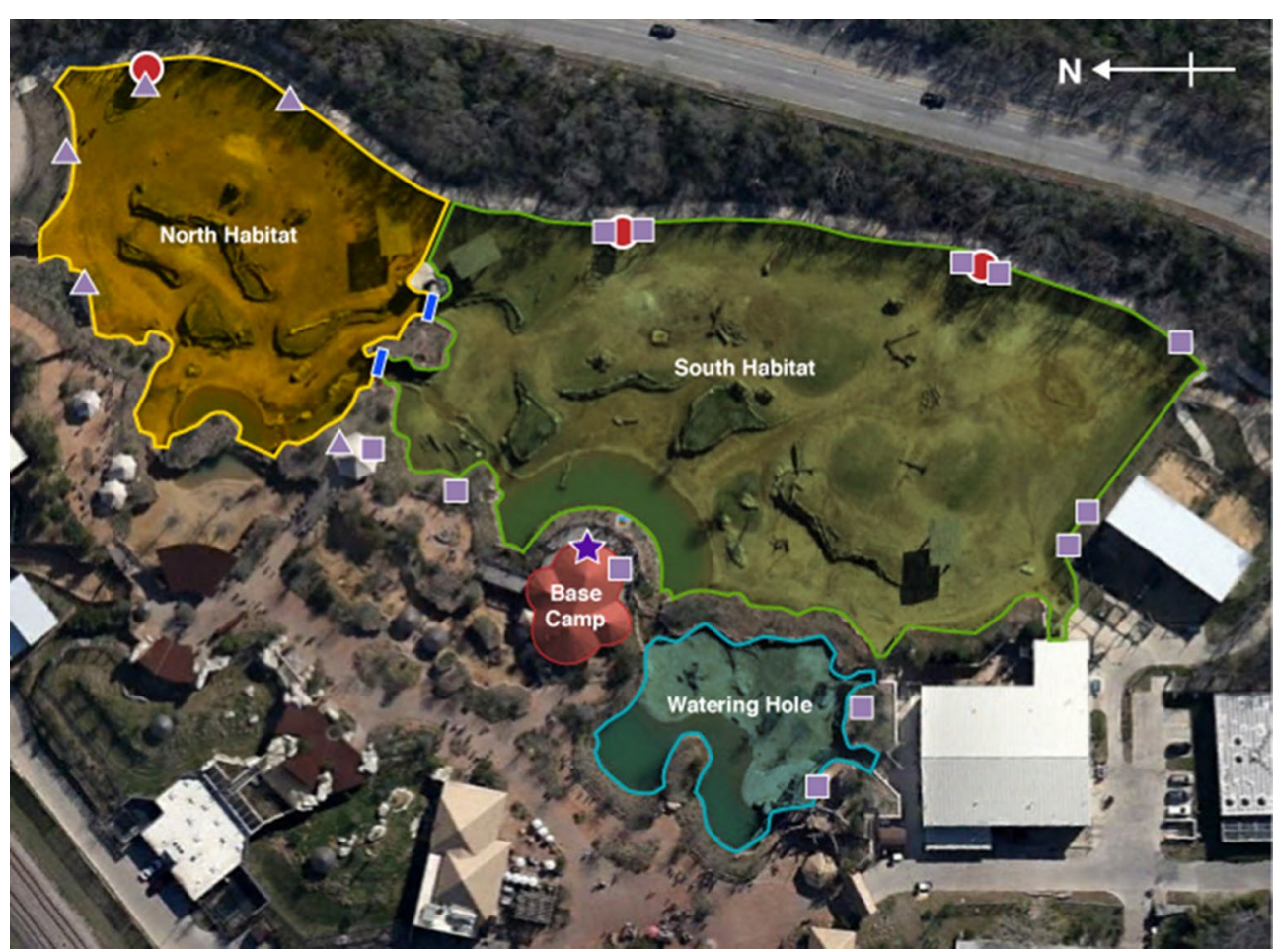

Fig. 1 Aerial view of the two larger yards (North and South Habitats) and the smaller yard (Watering Hole) in relation to the Base Camp interpretive center that houses the master anchor (indicated by a star). Two gates, illustrated with blue rectangles, connect North and South Habitat. Readers for North Habitat are indicated by triangles, while readers for South Habitat/Watering Hole are indicated by squares. Red circles show the locations of the three observation towers accessible to zoo personnel. Base map @2016 Google

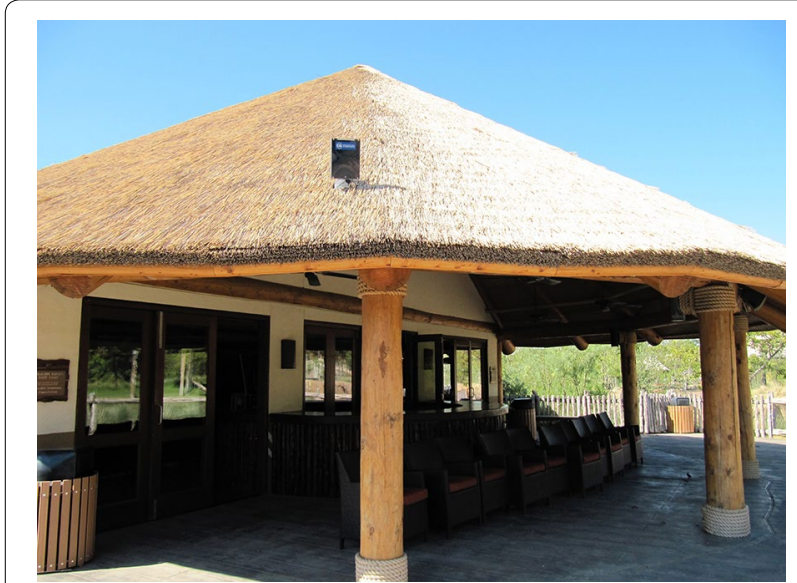

Fig. 2 A slave anchor installed on the roof of the Base Camp interpretive center

gambensis). Several days per week, the gates between the two exhibit spaces are opened to allow the various species to explore the entire exhibit and interact with each other. DZ staff have observed that the animals are more likely to randomly distribute themselves and use the entirety of the habitat as they actively explore this shared space. Zoo visitors have expressed their appreciation of seeing the animals exhibiting naturalistic behaviors among other species; no other zoo in the USA currently exhibits elephants with giraffe or other species. Elephants also occasionally have access to a nearby smaller yard (the Watering Hole) to give visitors a closer view of the elephants, but this yard is not accessible to other species.

Radio and satellite telemetry collars have been used for decades to monitor the movement of wild African savanna elephants [1-3]. In the field, this requires the use of a potentially stressful and dangerous procedure to sedate the animal to apply the collar to their neck. Since elephants live in social groups $[1,4]$ there is also potential danger of interfering with other animals besides the targeted individual. While passive RFID tags (e.g., microchip implants and PIT tags) have been used for a number of years in animals to identify individual pets and livestock as they pass a nearby receiver or scanner, active RFID tags have typically only been used for asset tracking and inventory monitoring of inanimate objects. To our knowledge, this is the first use of powered, active RFID tags to monitor real-time individual animal movement and space use at a zoo. 
To monitor movement of elephants in environments such as zoos, attempts have been made to adapt technologies that include global positioning systems (GPS) in neck collars $[5,6]$, and GPS and/or accelerometer units in bracelets $[6,7]$. Due to DZ's animal management restrictions and staff safety concerns, we were unable to use GPS neck collars for tracking elephant movement. Miller et al. [7] found that GPS leg bracelets only collected useful data reliably under sunny conditions. While the weather is often favorable in Dallas, we wanted to be able to collect data regardless of the weather or sky conditions. Berkovich (personal communication) reported losing GPS signals at San Diego Zoo near solid structures (e.g., solid walls) and inside buildings. The elephant habitat at DZ has varying topography and numerous solid structures throughout that could possibly interfere with GPS signals. Application of any GPS technology would also be restricted to outdoor spaces, preventing the ability to collect data on a 24-h basis-RFID anchors/readers can be added inside buildings for continuous coverage both indoors and outdoors.

After investigating numerous technology alternatives, we designed a bracelet that holds an active RFID tag that can be readily and innocuously fastened around an elephant's front leg. The tag continuously reports an elephant's location and movement in near real-time under various environmental conditions. The tags were reported by the manufacturer to achieve a location accuracy of about one meter outdoors; this was subsequently confirmed through the course of the development of the pilot system (Scott, unpublished data).

The purpose of ROAM at DZ is to collect locationally accurate data for the continuous digital observation of the elephants under different environmental conditions as they move throughout the exhibit, interact with each other, other animal species, permanent exhibit features (e.g., shade structures, water pools), and temporary exhibit features (e.g., food, mud wallows, movable logs) intended to stimulate the elephants' behavior. The data are analyzed to provide different audiences (e.g., husbandry staff, researchers, visitors) with insights they can use for their respective roles. Here, we describe the specifications and functionality of ROAM at DZ.

\section{Methods}

\section{Hardware}

ROAM uses hardware and software components produced by Convergence Systems Limited (CSL; Hong Kong) that include a wide beamwidth master anchor with $180^{\circ}$ antenna angle (CSL CS5114; Fig. 3), a series of wide beamwidth slave anchors with $180^{\circ}$ antenna angle (CSL CS5112) and narrow beamwidth slave anchors with $80^{\circ}$ antenna angle (CSL CS5111) distributed around the

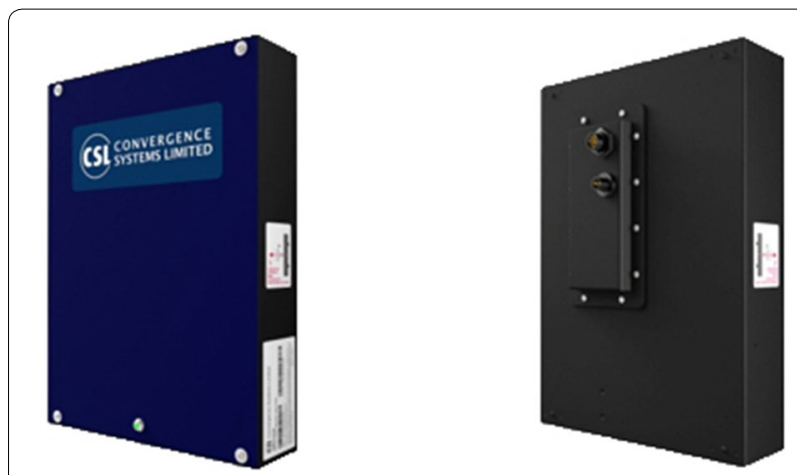

Fig. 3 CS5113/4 front view (left) and rear view (right) of the master anchor connected to the data-collecting computer

perimeter of the total exhibit area to maximize coverage and eliminate dead zones. Each anchor is in a watertight enclosure measuring $29 \times 21.2 \times 8 \mathrm{~cm}$ and weighing $1.5 \mathrm{~kg}$. Given that the tags require line of sight with at least three readers to enable the software to accurately perform the location and motion calculations, placement of the readers required initial adjustment to overcome the curvilinear design of the exhibit and minimize interference from retaining walls and grade changes.

Ten of the readers (including the master anchor) are powered directly by a $120 \mathrm{~V}$ electric conduit using a 12 V DC 1500 mA AC power adapter. Eight readers are remotely located and require electrical power provided by solar panels and backup batteries (BatteryMINDer Solar Charging System 12 V, 15 W panel, model SCC015; VDC Electronics, Inc., Englewood, NJ) maintained by three $12 \mathrm{~V} / 7 \mathrm{~h}$ sealed lead-acid batteries connected in parallel (Enercell; radioshack.com). Each tag is powered by three rechargeable NiMH AAA batteries (Sanyo Eneloop $800 \mathrm{mAh}$ ) typically yielding at least $48 \mathrm{~h}$ of battery lifespan at the current configuration settings. The master anchor is connected via Ethernet cable to a portable computer in the nearby Base Camp interpretive building that allows for a live feed display of the data on a large monitor for zoo visitors and interpretive staff. All of the slave anchors communicate with the master anchor wirelessly. The slave anchors (CSL P06, P12) are installed strategically around the perimeter of the entire exhibit space on exterior fencing supports and attached to the observation towers with mounting brackets to provide higher elevations for the readers to cover greater coverage of the exhibit space (Figs. 1, 2). The anchors are oriented in portrait mode to maximize the horizontal (azimuth) antenna coverage to compensate for the change in grade from the front to the back of the exhibit space. The down-tilt angle for each reader is approximately $45^{\circ}$ from horizontal to maximize antenna coverage. 
Weather permitting, each elephant wears an active RFID asset tag (CS3151TC; Fig. 4) with a unique serial number anytime they are outside day and night, up to $22 \mathrm{~h} / \mathrm{d}$. When a tag is first registered with the system, it is assigned a logical name (e.g., the first two letters of the assigned focal elephant), an average height for the bracelet and tag on the wrist of the elephant $(0.5 \mathrm{~m})$, and a maximum velocity allowed for the tag $(2.5 \mathrm{~m} / \mathrm{s})$. At the time of this publication, 6 adult female African elephants at DZ have been outfitted with the soft bracelets.

Prior to opening the Giants of the Savanna exhibit in 2010, each elephant was conditioned to present each manus or pes through an opening in a training wall in the elephant barn for routine husbandry. All elephants had prior training wearing chain bracelets for their transport to this new exhibit (a common safety practice for captive elephants); wearing a soft bracelet outfitted with an RFID tag was assumed to be an easy next step for the elephants. Each bracelet (Fig. 5) is custom-designed by Delta Rigging (Arlington, TX) based on the circumference of the carpal region of each elephant. Bracelets are constructed of polypropylene webbing wrapped with cordura. They contain a pouch midway along the bracelet to hold a water-resistant box (S3 model T1000, Fort Collins, CO) that contains the RFID tag. The weight of the bracelet, tag, and batteries is approximately $1.5 \mathrm{~kg}$ and does not appear to affect the behavior of the elephant (Scott, unpublished data).

\section{Software data settings}

Communication between the tags and the readers is based on time-division multiplexing that allows the tags to communicate with the readers sequentially according

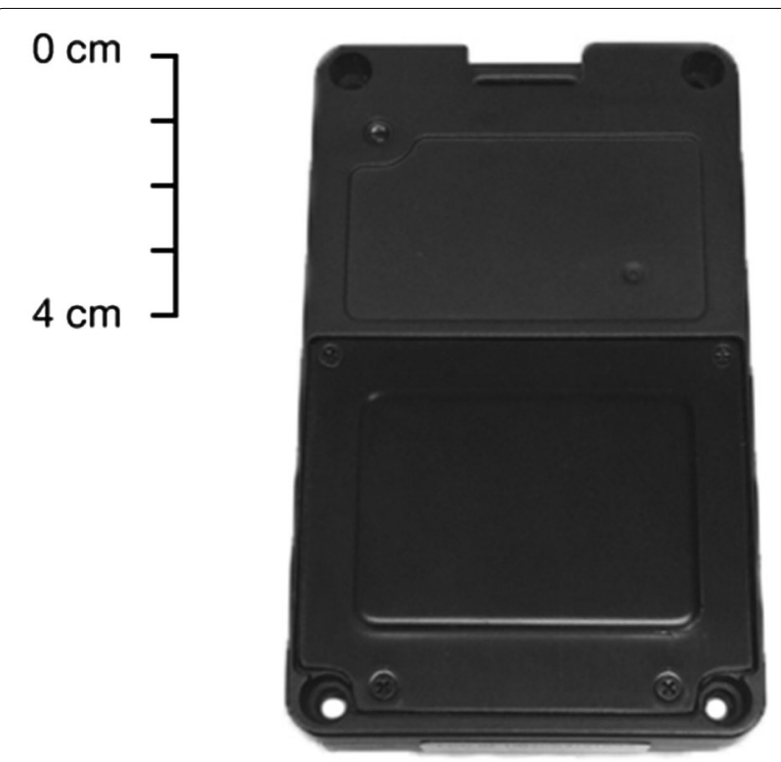

Fig. 4 CS3151TC asset tag to their system-assigned time slot within the ranging cycle. The current system configuration has each tag transmitting a wireless signal once every $6 \mathrm{~s}$, as assigned by the user. This frequency of signal transmission allows up to 10 tags to operate simultaneously. As the number of tags increase, the cycle time must increase to accommodate the volume of data being transmitted. If tags were placed on all $\sim 50$ individuals of all species in the mixedspecies habitat, the cycle time would need to be adjusted to every 7-8 min to collect all of the data. An important consideration when determining an appropriate cycle time is that locational accuracy of a tag increases as the frequency of data collection increases, but at the expense of decreasing the lives of the tag batteries. Within the 6-s cycle, there is also a time allocation for the registration of each new tag so that they are added to the system dynamically without any configuration upfront or operator involvement.

\section{Data collection}

The master anchor itself is located on the roof of the Base Camp building to provide it with optimal line-ofsight access to a majority of the slave anchors. For the slave anchors that do not have line of sight to the master anchor, the system is designed so that slave anchors can relay their signal to another slave anchor that does have direct line of sight to the master anchor. The range of the readers is reported by the manufacturer to be up to $200 \mathrm{~m}$ outdoors. This was subsequently confirmed through the development of the pilot system (Scott, unpublished data). The habitat is approximately $230 \mathrm{~m}$ at the widest diagonal but less than $75 \mathrm{~m}$ wide in most areas. The coordinates of each slave anchor (relative to the master anchor at the origin) were manually transcribed into the system so that they can be seen visually on an aerial view of the exhibit in relation to other readers and to the location of the tags. The height of each slave anchor around the perimeter of the habitat is then defined relative to the lowest point in the exhibit (i.e., the pool). The RTLS system uses a time-of-arrival (TOA) ranging technique to estimate the distance between tags and multiple readers. This method uses the time required for the signal emitted by a tag to travel (i.e., the propagation delay) to a reader to calculate the distance between the tag and a given reader. The system operates within the $2.4 \mathrm{GHz}$ ISM frequency band. As the traveling signal approaches the speed of light, the tag location [i.e., the $(X, Y)$ coordinate relative to the master anchor at the origin] can be estimated by the locational computation engine in the software from the elapsed time to each reader of a known location. Raw location data are then saved on the personal computer at Base Camp directly connected to the master anchor for storage, analysis, and display. 


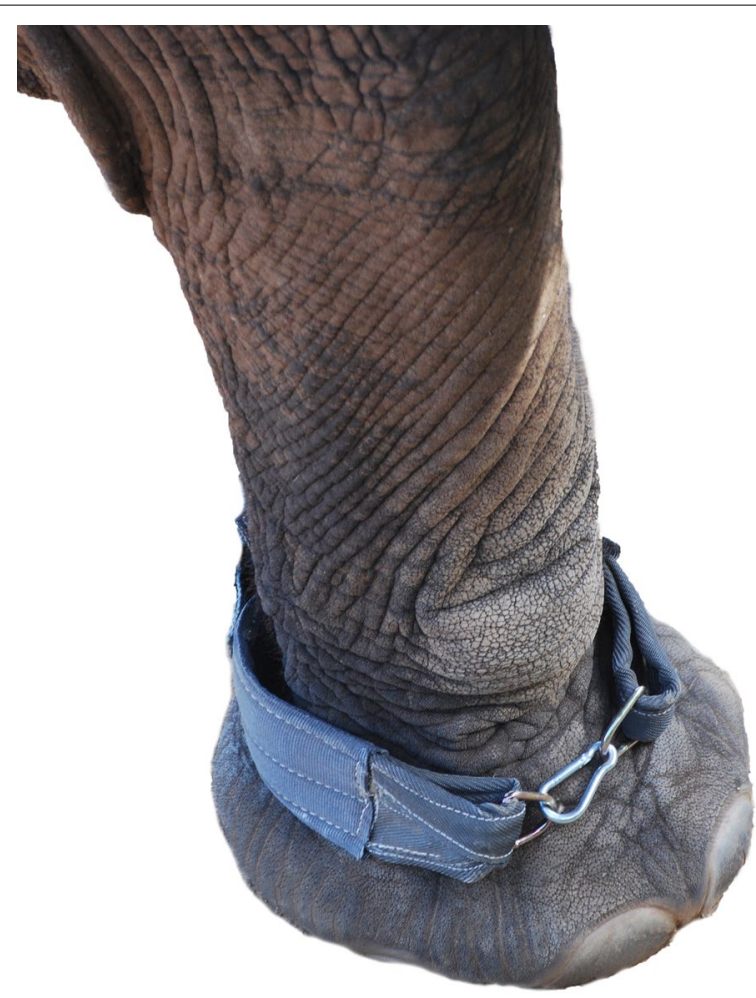

Fig. 5 RFID tag in bracelet on elephant manus

\section{Filters}

The filters built into the software are settings that can be adjusted to instruct the system to accept data points satisfying specific criteria while dismissing any unacceptable data points. Such filtering ensures the capture and analysis of only high-quality data. Signal "noise" happens under a number of circumstances, such as when a signal from a tag is not registered by at least three readers (which puts its location in doubt), or when tag signals reflect off hard surfaces such as retaining walls (causing instability in the tag's location). Tag signal quality can be improved by adjusting the filter settings. The most critical filter is the Rolling Window Average. It calculates the location of the tag based on the moving average of a user-defined number of previous tag data points (e.g., the last 3 locations). This setting is particularly effective at minimizing noise in areas prone to a high percentage of signal reflections and distortions of a tag's perceived location contributing to an increase in latency of tag signal detection by the readers when the elephants are in motion. The Maximum Velocity Filter (in a user-defined number of meters/second) is a location-based filter setting for limiting the amount of change in a tag's location within $1 \mathrm{~s}$. Erroneous data are eliminated if a tag signal appears to be moving faster than allowed within $1 \mathrm{~s}$ (e.g., $\left.v_{\max }=2.5 \mathrm{~m} / \mathrm{s}\right)$. A Permitted Boundary Filter allows a user to define the boundary of an area where tag signals are permitted; data points found outside of the user-defined exclusion area are eliminated. This filter eliminates signals that are not relevant to the various calculations.

\section{Results and discussion}

In real-time, ROAM records the coordinates of each elephant every $6 \mathrm{~s}$, displays their location within the habitat, and shows which anchors are detecting the tag's signal (Fig. 6). The distance between the tag and the anchor determines the radius for each ranging circle. The intersection of multiple ranging circles displays the location of the tag/elephant. Location data are autoarchived as a CSV file for further analyses and comparison with additional variables. Preliminary data analysis has shown significant differences in rates of travel relative to environmental temperature [8]. Habitat use is also displayed visually in real-time as a single hue progression choropleth map with highly frequent areas displayed in darker shades and less frequented areas in lighter shades (Fig. 7). This infographic has been a helpful tool for elephant husbandry staff to ensure the elephants are engaged with their environment and using the entirety of the habitat available to them both day and night. The Giants of the Savanna exhibit was designed to provide elephants and other species the opportunity to express naturalistic behaviors: Feeders have been strategically placed to encourage active foraging, multiple water features promote self-maintenance behavior, and varying terrain grades stimulate movement. By monitoring exhibit use and managing animals to utilize the entirety of their available space, there is a greater likelihood that these behaviors will occur [9].

Real-time imagery allows animal keeper staff to remotely know where all of the elephants are in the habitat from anywhere in the world-whether from inside the adjoining elephant barn, across the zoo in an office, or even offsite. There is a growing trend to manage the lives of captive animals around-the-clock, even when husbandry staff are not present on grounds (e.g., automatic feeders permit food to be gradually dispensed over a long period). A system such as ROAM allows for the behavioral effects of these practices to be objectively monitored and quantified for analysis by husbandry staff and researchers. Furthermore, the behavioral preferences of individual elephants are evident, with most exhibiting increased use of areas where environmental features (such as mud wallows and shade structures) have been provided, and some taking advantage of 


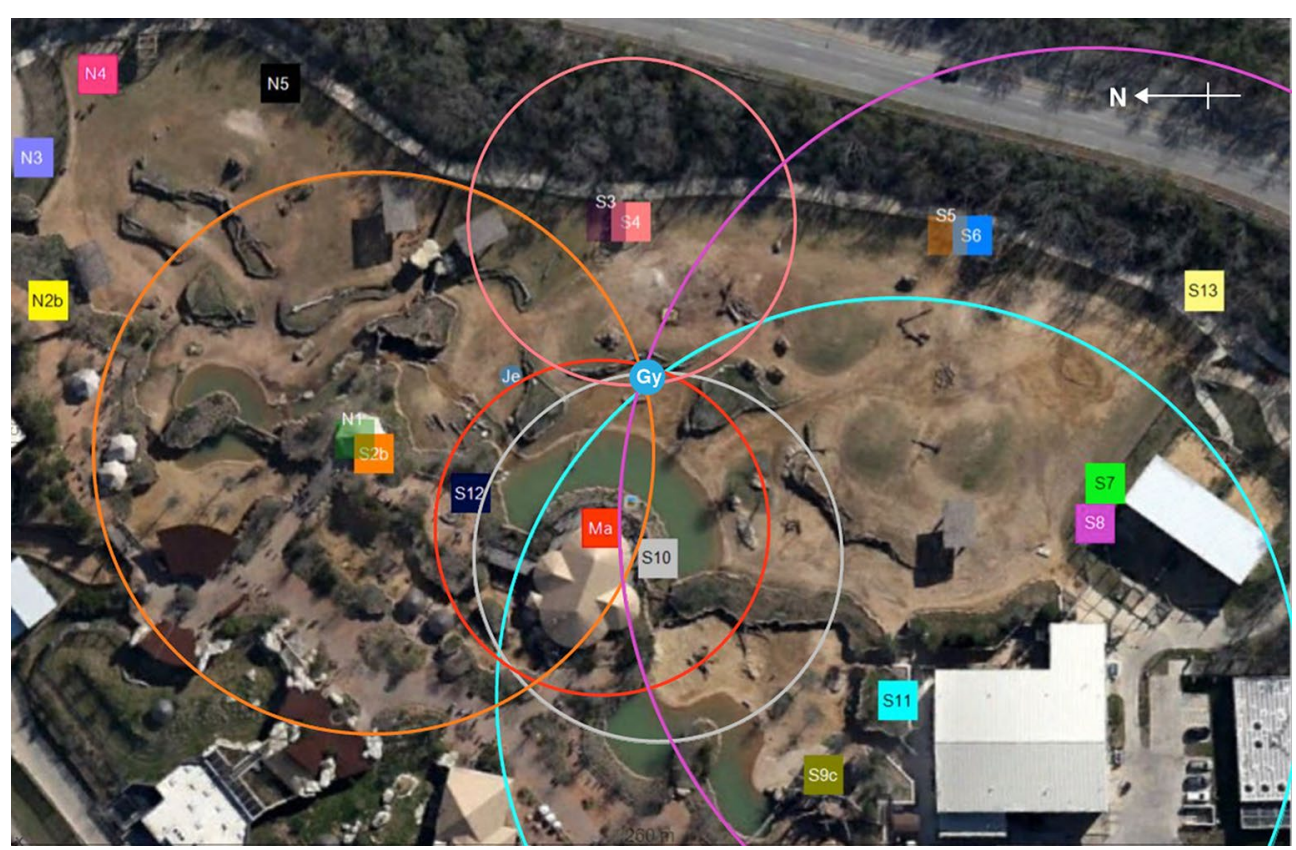

Fig. 6 Two elephants in the South Habitat (Je "Jenny"; Gy "Gypsy") with ranging circles from the six readers multilaterating on Gypsy's tag

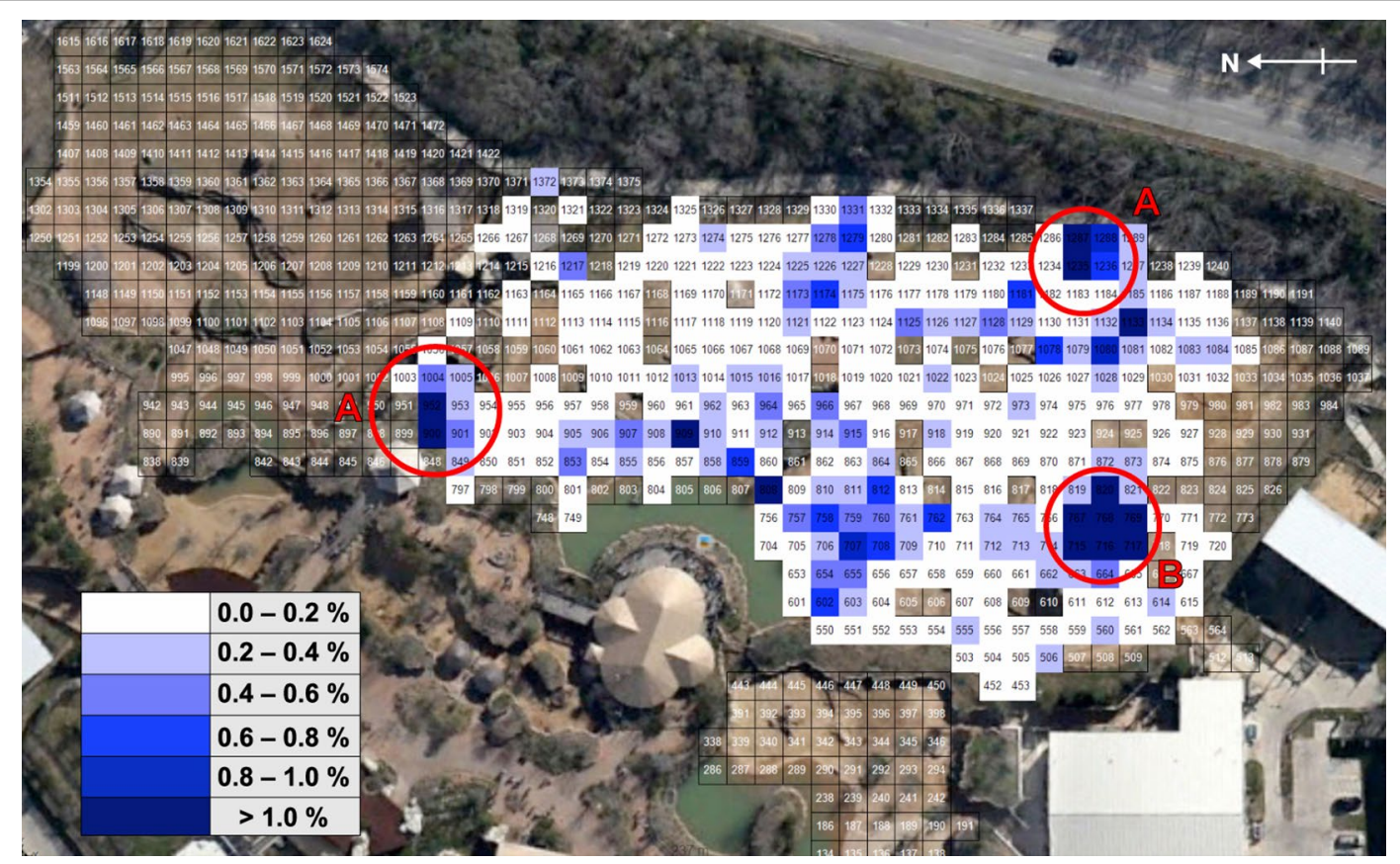

Fig. 7 A single hue progression choropleth map generated from 3830 position points representing South Habitat use for one elephant ("Congo") over 8 daytime hours on July 20,2015. Each square in the grid depicts a $5 \mathrm{~m} \times 5 \mathrm{~m}$ area of the habitat. Note the circled areas of high use (A elevated hay and browse feeders; $B$ shade structure). Base map $\odot 2016$ Google 
more transient features, such as browse piles or elevated hay nets (Fig. 7). These individual differences in behavior and exhibit use are important to consider in an animalbased welfare assessment approach [10].

Besides identifying areas of high interest within the habitat, the routes that the animals use throughout Giants of the Savanna on a given day are generated by ROAM in real-time and are autoarchived as image files periodically in user-defined time intervals (Fig. 8). These images are routinely reviewed by keeper staff for elephant husbandry purposes; any deviations in an elephant's normal area use or movement could be indicative of a medical issue (e.g., foot or joint issues) or social changes in group dynamics (i.e., avoidance of or attraction toward other individuals) and could then be addressed by keeper staff. As an example of the health monitoring benefits of ROAM, the walking rates of one geriatric elephant were compared closely each month during her last 2 years; these measures informed the care plans developed by husbandry, nutrition, and veterinary specialists.

ROAM has proven to be effective for collecting highquality location data necessary for continuously monitoring elephant movement, social associations, and exhibit use. Benefits to husbandry staff and managers have been described, but data are valuable to diverse audiences within and outside DZ. The mission of DZ is to conserve wildlife and inspire a passion for nature among its guests, and zoo visitors have been shown to exhibit more interest in captive animals that engage in active behaviors [11, 12]. In this way, ROAM equips elephant husbandry staff with the knowledge to effectively increase the behavioral diversity of the animals under their care; visitors that observe behaviorally dynamic animals are likely more receptive to educational and conservation-based material. We are also currently developing platforms and visual analytics (displaying individual elephant movement and social data with environmental variables and other factors) for visitors to interact with the data, further fostering public appreciation for nature, science, and conservation.

The management potential of ROAM at DZ and beyond is likely still to be fully realized, and future manuscripts will present more detailed findings of results. With ROAM, we have already shown that ambient temperature influences elephant movement on an individual basis, and we are working to better understand the influence of other environmental variables (social access, space availability) and health measures (weight, diet) on movement and behavior. We are also interested in quantifying the behavioral benefits of the Giants of the Savanna exhibit to our elephants, and this technology will soon be expanded to include other species (e.g., giraffe). Using ROAM under a theoretical approach to behavior, we would also like to better understand how

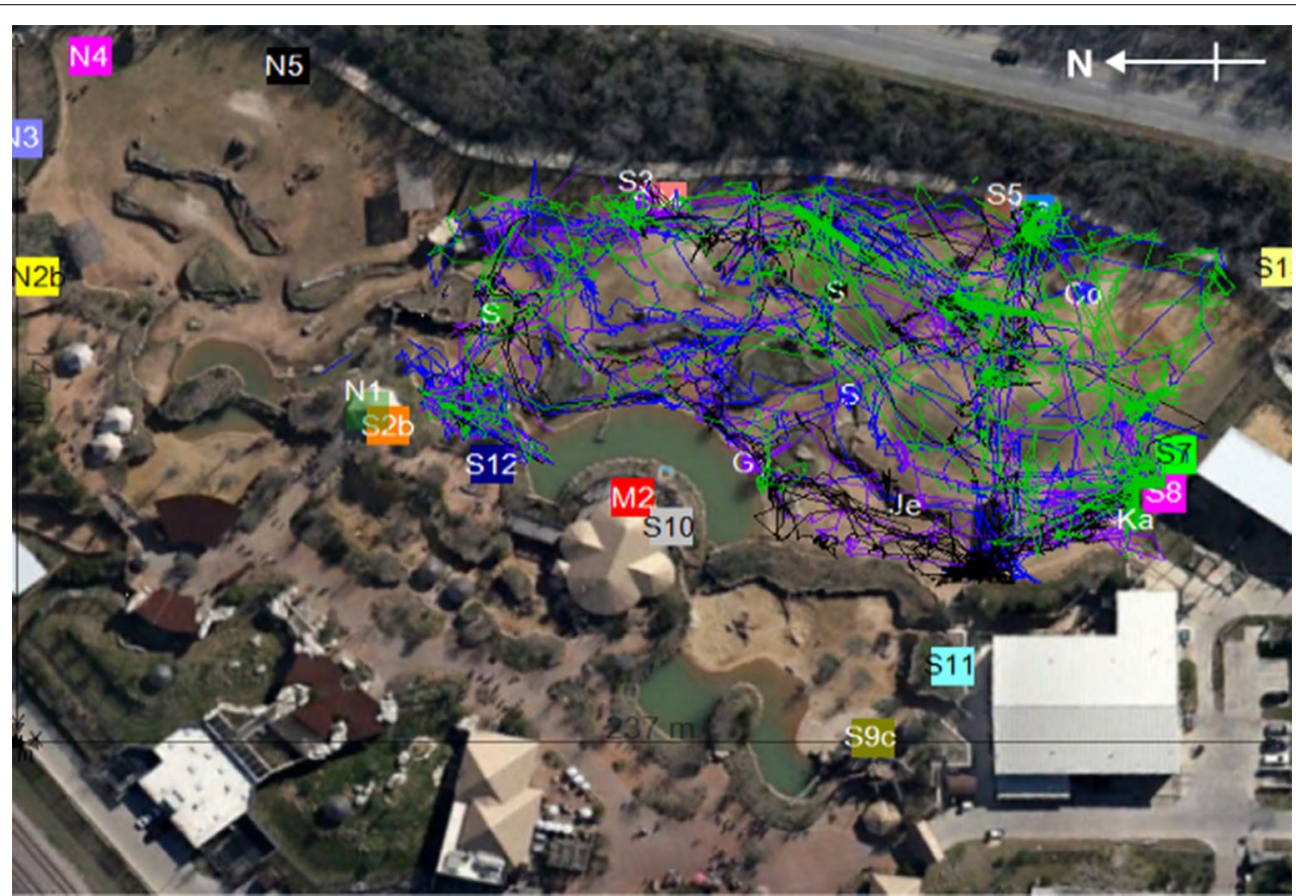

Fig. 8 Trails of four elephants (Co "Congo" in blue, Gy"Gypsy" in purple, Je"Jenny" in black, Ka "Kamba" in green) in the South Habitat over 8 daytime hours from January 14, 2016. Base map @2016 Google 
elephants interact with each other and how these interactions change over time; ROAM currently records the distance between tags at each time point, and we can couple these data with live behavioral observations. In a more applied sense, a growing database will be especially useful in describing the implications of expected (exhibit modifications, animal transfers, seasonal cycles) and unexpected (animal losses, social interactions) changes. More broadly, these findings will likely develop into specific tools to inform animal welfare and exhibit design. As mentioned previously, this is the first time to our knowledge that active RFID has been used to monitor the movement of zoo-housed animals. This project involved troubleshooting that may be unique to this or similar applications, and future applications with different species and institutions will inevitably eliminate or streamline problems with establishing a system like ROAM.

\section{Conclusions}

The RTLS pilot system at DZ is the first-ever use of active RFID technology to remotely and unobtrusively observe animal behavior patterns in a zoo exhibit $24 \mathrm{~h} / \mathrm{d}$. It has proven to be a successful tool for efficiently collecting high-quality, frequent data remotely throughout the day and night. Active RFID technology, developed for inventory tracking, is a valuable tool for monitoring the movement and space use of animals in human care. Using active RFID tags has allowed caregivers and researchers a view into how the elephants are interacting with each other and utilizing the space provided to them at DZ. Gathering data in near real-time to monitor the behavior and exhibit use of animals under human care is important for assessing their physiological, biological, and social needs. We suggest coupling RFID data with live behavioral observations, hormonal data, and other health measures to provide a complete depiction of animal welfare. Analysis of such data will provide a range of insights valuable to audiences including animal keepers, management staff, and zoo visitors. A system that continuously collects data provides a more accurate representation of animal behavior than periodic sampling observation by caregivers or researchers, whose presence can also affect the behavior of the animals. As the social dynamics change with evolving elephant herd dynamics, monitoring social associations day and night can be a helpful tool for animal care staff to know how animals are relating to one another in a provided space even in the absence of caregivers. The system represents the first phase of a multifaceted project that will add capabilities and functionality to improve animal care and wellbeing for additional species and at other facilities.

\section{Abbreviations}

CSL: Convergence Systems Limited; DZ: Dallas Zoo; GPS: global positioning system; ROAM: Real-Time Observer of Animal Movement; RSSI: received signal strength indicator; RTLS: Real-Time Location System; RFID: Radio Frequency Identification; TOA: time of arrival; ISM: industrial, scientific and medical.

\section{Authors' contributions}

NS implemented the system, was the onsite troubleshooter of both software and hardware, and drafted the manuscript. BH conceived of the study and participated in early installation of hardware. CAL significantly contributed to the manuscript, assisted with preliminary data analysis to confirm the system was operational, and produced the figures. CL, AL, and LC developed the site manager, created the filters, and updated the software as requested by NS and $\mathrm{BH}$. All authors read and approved the final manuscript.

\section{Author details}

${ }^{1}$ Conservation and Education, Dallas Zoo, 650 South RL Thornton Frwy, Dallas, TX 75203, USA. ${ }^{2}$ Animal Performance Systems, POB 11804, Bainbridge Island, WA 98110, USA. ${ }^{3}$ Department of Biology, Western Kentucky University, 1906 College Heights Blvd, Bowling Green, KY 42101, USA. ${ }^{4}$ Convergence Systems Limited, 20/F Chung Nam Building, No. 1 Lockhart Road, Wanchai, Hong Kong.

\section{Acknowledgements}

We would like to thank the Dallas Zoo elephant care team for their support and participation in this project, and the Zoo's electricians and IT team members for providing technical support. We also thank Dr. Lynn Kramer, Vice President of Animal Operations and Welfare, Dr. Patricia McGill, Vice President of Conservation and Education, and Karen Gibson, Savanna Curator and Elephant Manager, for supporting this research. The authors would also like to thank an anonymous reviewer for helping improve the manuscript.

\section{Competing interests}

The authors declare that they have no competing interests.

\section{Ethics approval and consent to participate}

Animals were not subjected to unnecessary pain or distress for this project.

\section{Funding}

This research was funded substantially by Dallas Zoological Management, Inc., with additional funding provided by Animal Performance Systems.

Received: 2 March 2016 Accepted: 21 July 2016

Published online: 02 August 2016

References

1. Douglas-Hamilton I. On the ecology and behaviour of the African elephant [PhD]. Oxford; 1972.

2. Evans KE. The behavioural ecology and movements of adolescent male African elephant (Loxodonta africana) in the Okavango Delta, Botswana [PhD]. University of Bristol; 2006.

3. Wittemyer G. The socio-ecology of the African elephant (Loxodonta africana) [PhD]. University of California, Berkeley; 2005.

4. Buss IO. Some observations on food habits and behavior of the African elephant. J Wildl Manag. 1961;25(2):131-48.

5. Leighty KA, Soltis J, Wesolek CM, Savage A, Mellen J, Lehnhardt J. GPS determination of walking rates in captive African elephants (Loxodonta africana). Zoo Biol. 2009;28:16-28. doi:10.1002/zoo.20199.

6. Rothwell ES, Bercovitch FB, Andrews JRM, Anderson MJ. Estimating daily walking distance of captive African Elephants using an accelerometer. Zoo Biol. 2011;30:579-91. doi:10.1002/zoo.20364.

7. Miller L, Andrews JC, Anderson M. Validating methods to determine walking rates of elephants within a zoological institution. Anim Welf. 2012;21:577-82. doi:10.7120/09627286.21.4.577.

8. Scott, NL, LaDue CA. Monitoring locomotion and exhibit use in zoohoused African elephants using RFID tags. In: Animal Behavior Society conference, Princeton; 2014. 
9. Swaisgood RR, Shepherdson DJ. Scientific approaches to enrichment and stereotypies in zoo animals: what's been done and where should we go next? Zoo Biol. 2005;24:499-518. doi:10.1002/zoo.20066.

10. Whitham JC, Wielebnowski N. Animal-based welfare monitoring: using keeper ratings as an assessment tool. Zoo Biol. 2009;28:545-60. doi:10.1002/zoo.20281.
11. Margulis SW, Hoyos C, Anderson M. Effect of felid activity on zoo visitor interest. Zoo Biol. 2003;22:587-99. doi:10.1002/zoo.10115.

12. Moss A, Esson M. Visitor interest in zoo animals and the implications for collection planning and zoo education programmes. Zoo Biol. 2010:29:715-31. doi:10.1002/zoo.20316. 